\title{
The influence of direct and indirect semantic contexts on binocular-rivalry resolution*
}

\author{
ROGER B. HOWARD \\ Colgate University, Hamilton, New York 13346
}

\begin{abstract}
Sixteen Ss were asked to identify an unknown word as the E gradually increased the illumination behind it. Unknown to the $\mathrm{S}$, there were two words superimposed stereoscopically (e.g., BAT/BAG) so that binocular rivalry (BR) was established between the last two letters. If the $B R$ stimuli were accompanied by a direct semantic context (e.g., T), the Ss reported the related alternative significantly more often than the other. Indirect semantic contexts (e.g., BALL) had no effect on BR resolution. Most of the Ss reported that they did not see the BR letters as clearly as the other material on the card, even after resolution had occurred, and all Ss failed to realize that there were two alternatives until the luminance from the surface of the card had increased to at least $0.5 \mathrm{fc}$. These results, in conjunction with other information available on the neuropsychology of stereopsis and BR, suggest that direct semantic contexts produce perceptual biases by facilitating the suppression of one alternative by the other. Indirect contexts probably produce response bias and little, if any, perceptual suppression.
\end{abstract}

Rommetveit, Toch, and Svendsen (1968) have proposed a method for studying the effects of semantic contexts upon perception. They used a tachistoscope to present two pairs of stereoscopically superimposed words. The upper pair of words was the same (e.g., DEVIL/DEVIL) and so fused into a single percept. The lower pair of words was the same except for one letter (e.g., HELL/SELL) thus producing binocular rivalry (BR). If the upper word was related to one of the alternative lower words, the Ss tended to resolve the BR in favor of that alternative.

The possibility of using BR resolution as a measure of semantic context effects is very appealing. Unfortunately our pretest Ss found it difficult to converge their eyes and maintain convergence long enough for the stimuli to be presented. There was also a tendency for the Ss to perceive both alternatives at some point in the experiment. Finally, it is conceptually difficult to separate perceptual from response biases in Rommetveit's design. Signal detection methods such as those used by Herzog (1971) are not applicable because detection of the "correct" BR stimulus is confounded with the overly simple task of detecting the difference between the context materials. Since Herzog's data suggest that tachistoscopic studies probably include both perceptual and response biases, it is doubtful that Rommetveit's procedures will produce unambiguous information.

In the research reported below, pairs of stimuli similar to Rommetveit's were placed in a light-tight box, and the illumination behind the stimuli was gradually increased by a method of limits procedure until the $S$ could report the context material and one of the two BR stimuli. Our pretest results suggested that if an $S$ could

\footnotetext{
*This research was supported in part by grants to the author from the Colgate Research Council and the Sloan Foundation. It was completed while the author was a trainee at Duke University in the Sciences Related to the Nervous System.
}

converge his eyes on a fixation dot at the beginning of the series, then he never had focusing problems throughout the experiment. Further, once an $\mathrm{S}$ had formed a correct hypothesis about the ambiguous word, the illumination could be increased until he was sure of his response, and yet he could not detect the alternative letter.

It seems reasonable to distinguish between at least two kinds of semantic contexts. A direct semantic context is one which provides the missing information directly. Herzog (1971) used the stimulus itself as the primary context in a repeated exposure paradigm. An indirect semantic context is one which provides the missing information indirectly. Rommetveit studied indirect context effects when he presented material (DEVIL) which was related to one of the alternatives (HELL rather than SELL). Since direct contexts produce the information needed to resolve the rivalry with the least ambiguity, they should have a stronger effect on perception than indirect contests.

\section{METHOD}

The Ss were eight male and eight female undergraduates who volunteered for the experiment; all had normal acuity and were right-eye dominant.

The BR stimuli consisted of eight pairs of CVC words which were selected so that (a) the meanings of the two words in a pair were not closely related, and (b) no two pairs had the same last two letters. The direct context was the last letter of one of the BR stimuli. The indirect context was a word related to one of the alternatives in the pair but not the other. The materials were typed on $5 \times 7$ in. cards, using a bulletin typewriter.

The Ss were seated in front of the apparatus and read the following instructions: "The procedure is very simple. I'm going to show you some cards with verbal material on them in this box. There are two kinds of cards. One kind has two words on it, one above the other. In some cases the first word will be related to the second one. The other kind of card has a letter above a word. In some cases the letter will be the same as the last letter of the word. Before each trial, I will tell you what the upper word or single letter is. Then I will turn out the light. You look 
through the viewer here in front of the box while I gradually increase the light. The first thing you will see is a dot of light. If you see two dots tell me immediately. The dot will be to the right of the upper word or letter-the one which I will have given you. The unknown word will always have three letters.

"The main idea is to tell what the material on the card is at the lowest light level possible. In each case try to make out the word or letter which I have given you first and then the unknown word. The word or letter which I give you will always be the upper one on the card, but make sure you see it anyway.

"If your answer is wrong or if you are not sure of the stimuli I will increase the illumination until you are correct and sure. Between trials, please look away to the right and keep your eyes closed."

The stimuli were presented in a box painted flat black both inside and out with a stereo viewer mounted so that it projected through the front. The $E$ increased the illumination behind the cards by turning a potentiometer connected in series with a $6-\mathrm{V}$ battery and a small clear bulb positioned exactly behind the intersection of the horizontal and vertical midlines of the card. Each increment was $0.1 \mathrm{~A}$. The maximum luminance from the surface of the card was $0.5 \mathrm{fc}$.

Each $\mathrm{S}$ was shown two practice cards with no BR between the two pairs of superimposed words, followed by eight experimental cards containing BR and context materials. There were three variables which were counterbalanced: direct or indirect contexts; related to the first or second words of each BR pair; presented to the left or right eye. All combinations of these variables were presented to each $S$, and the serial order of each combination was counterbalanced with each sex. The mapping of specific context-BR stimuli configurations into this design was essentially random, with the constraint that each combination appear equally often and the serial position of each combination be the same across the eight Ss of each sex.

On the last card, the illumination was increased to its maximum even if the $S$ was sure of the word at a lower illumination. If he failed to notice the second alternative for the last letter, the top was taken off the box and the room lights were turned on. The $\mathrm{S}$ was then questioned in detail about his experiences in the experiment.

\section{RESULTS}

Since the mean number of context-related stimuli reported by male and female Ss were virtually identical (3.3 and 3.4 for direct contexts; 2.3 and 2.0 for indirect contexts), the data were collapsed across the sex variable. The Ss saw $83 \%$ of the context-related words of the BR pairs within a direct semantic context $(\mathrm{t}=6.894$, $\mathrm{df}=15, \mathrm{p}<.0005$, one-tailed) and $53 \%$ of the context-related words within an indirect semantic context $(\mathrm{t}=0.492, \mathrm{df}=15, \mathrm{p}>.20$, one-tailed $)$. The number of context-related words perceived within the direct context is also significantly greater than the number of such words seen within the indirect context $(\mathrm{t}=3.967, \mathrm{df}=15, \mathrm{p}<.005$, one-tailed $)$.

None of the Ss realized that there were two letters in the last position even when asked if they were sure of their responses. Twelve failed to perceive the alternative until the luminance from the card was increased to $0.5 \mathrm{fc}$. Four failed to see the alternative until the top of the box was removed and the lights were turned on in the room.

\section{DISCUSSION}

The data suggest that a direct context may resolve BR in favor of the context-related alternative. Response bias enters when the $S$ must guess because the stimulus does not provide sufficient information. All of our Ss reported that they were sure of their percepts on all trials and required no additional information (via increases in illumination). They were universally indignant when the $\mathrm{E}$ implicitly questioned their response on the last trial by continuing to increase the illumination beyond the point at which they were confident. It is very unlikely that response bias, as the term is currently used, can explain these results.

The data also indicate that indirect contexts probably contribute response rather than perceptual biases. The Ss' protocols showed that they often correctly guessed the relationship between the indirect context and the missing letters, but rejected that hypothesis in favor of the other alternative. This suggests that the association between the context and the related alternative was not strong enough to capture the percept. Since Rommetveit et al (1968) found that indirect contexts were effective in determining the S's responses, it seems likely that indirect contexts contribute response rather than perceptual biases. Ss make the most likely guess given the indirect context and the clear letters of the rivalry word, but when they are allowed additional information, the percept is determined by other factors.

Researchers who believe that context produces a perceptual bias have usually assumed that it heightens the sensitivity of the organism to the stimulus (cf. Haber, 1966). In contrast, the emerging physiological and psychological evidence indicates that $\mathrm{BR}$ resolution is mediated by inhibition. A large number of neurons in the striate cortex of many animals respond best to binocular input (Bishop, 1971). Monocular input produces weaker responses, and stimuli which would cause BR in humans produce inhibition of one response by the other. Wales and Fox (1970) have found analogous increments in detection thresholds for the suppressed field under BR conditions with human observers.

The data reported here support this model in two ways. Since the neurons in question respond optimally to binocular information, we would expect the nonrivalry letters to be seen with lower illumination than the BR materials. Further, since suppression of one field by the other produces weaker firing in the neurons mediating stereopsis, we would also expect that the percept of the resolved rivalry letter would be weaker than the letters around it. The data from this experiment indicate that the Ss always saw the nonrivalry materials before they saw the rivalry letters. Further, they frequently complained that the BR letters were less clear than the other letters in the same word or the context material, even after they were sure of their responses. Thus BR is probably resolved at the cost of perceptual clarity, and not through facilitation of the dominant stimulus.

\section{REFERENCES}

Bishop, P. O. Neurophysiology of binocular single vision and stereopsis. In R. Jung (Ed.), Handbook of sensory physiology. New York: Springer, 1971.

Haber, R. N. Nature of the effect of set on perception. Psychological Review, 1966, 73, 335-351.

Herzog, T. R. Effects of amount and kind of prior target-uncertainty reduction in the repeated presentations paradigm. Perception \& Psychophysics, 1971, 10, 273-277.

Rommetveit, R., Toch, H., \& Svendsen, D. Effects of contingency and contrast contexts on the cognition of words. Scandinavian Journal of Psychology, 1968, 9, 138-144.

Wales, R., \& Fox, R. Increment detection thresholds during binocular rivalry suppression. Perception \& Psychophysics, $1970,8,90-94$.

(Received for publication May 30, 1974.) 